\title{
Propagation of $T M$ surface modes in a taper slab waveguide with anisotropic metamaterials
}

\author{
Lu Fa Shen ${ }^{1}$, Jian Ping Xie ${ }^{1}$ and $\mathrm{Zi}$ Hua Wang $^{2}$ \\ ${ }^{1}$ Science College, Huzhou University, Zhejiang, 313000, P. R. China. \\ ${ }^{2}$ School of Communication and Information Engineering, Shanghai University, Shanghai, 200072, P. R. China. \\ E-mail: shenlufa@zjhu.edu.cn
}

ABSTRACT: The $T M$ surface mode propagation properties in a taper slab waveguide with an anisotropic metamaterial core and common material claddings are investigated. Through Maxwell's equations, the dispersion, reflection coefficient, transmission coefficient and power loss were extracted for the $T M$ surface modes. Then, some characteristic curves are plotted for different metal filling ratios or different frequencies. From these curves, it is found that: (1) as waveguide length $\mathrm{L}=7.7 \mu \mathrm{m}$, the reflection coefficient is near 0.99 ; (2) the transmission coefficient $\left|c_{t}\right|^{2} \leq 0.1$ or even $\left|c_{t}\right|^{2} \leq 0.05$; (3) the power loss $\Delta p / p \geq 0.98$ or even $\Delta p / p \geq 0.99$, which means extra strong reflection or extra big energy loss can exist in the taper waveguide. These new properties for $T M$ surface modes propagation will benefit us to design extra strong reflectors or novel absorbers in the future.

Key words: taper slab waveguide, anisotropic metamaterials, surface modes, power loss

\section{Introduction}

In 1968, Vesalago [1] predicted theoretically negative index in materials where the dielectric permittivity and magnetic permeability are simultaneously negative. This materials are known as metamaterials or left-handed material (LHM) because their electric field, magnetic field vectors and wave vectors are in keeping with left—handed system as electromagnetic waves propagate into it. However, his prediction cannot be turned into reality because of technology 
limited at that time.

Up to 2000, Smith et al. had made firstly the metamaterial in microwave band [2] and explored widely for the metamaterial waveguides [3-5]. With the development of science and technology, metamaterials may be constructed by alternating metal-dielectric multi-layer structures or combining metal nanometer wires with some dielectrics $[6,7]$.

In 2007, K. L Tsakmakidis predict that a taper metamaterial waveguide can stop the light [8] and that attracts much interest for researchers because of its potential application in light storage. Up to now, the tapered metamaterial waveguides have been widely studied both on simulations and experiments [9]. It is found that light cannot be stopped completely because the metamaterial has strong losses, and there is an extra strong reflection existing in a taper slab waveguide [10]. Based on above-mentioned investigations, many researchers have been beginning to design the extra strong absorbers through COMSOL software [11]. Although some significant results have been obtained, there is still a lack of systematic theoretical analysis.

In this paper, we study the propagation properties of the $T M$ modes in a taper slab waveguide which consists of a core layer of anisotropic metamaterials and cladding layers of common materials. From Maxwell's equations and the boundary condition (i.e., the tangential fields must be continuous between the layers of a slab waveguide), a dispersion equation for $T M$ surface modes is derived. Then, through mathematics calculation, the reflection coefficients , transmission coefficients and power loss are obtained, respectively. From the above equations, we plotted some corresponding curves, and found some new properties for $T M$ surface modes in a taper slab waveguide with anisotropic metamaterials.

\section{Dispersive equation and power flow for TM surface modes}


The schematic geometry of a three-layer slab waveguide with metamaterials in Fig. 1. The core layer (thickness $2 d$ ) is an anisotropic metamaterial with permittivity tensor $\stackrel{\leftrightarrow}{\varepsilon}\left(\varepsilon_{1 x}, \varepsilon_{1 y}, \varepsilon_{1 z}\right)$ and permeability tensor $\stackrel{\leftrightarrow}{\mu}\left(\mu_{1 x}, \mu_{1 y}, \mu_{1 z}\right)$, while the cover and substrate layers are the same common material with permittivity $\left(\varepsilon_{2}\right)$ and permeability $\left(\mu_{2}\right)$. It is known as a symmetric slab waveguide in which $T E$ and $T M$ modes can exist. Here, we mainly discuss the $T M$ modes and the $T E$ modes will be discussed elsewhere. For TM modes, based on Maxwell's equations, the electromagnetic field components $E_{x}, H_{y}$ and $E_{z}$ are satisfying the following equations in the core layer.

$$
\begin{aligned}
& i \beta E_{x}+\frac{\partial E_{z}}{\partial x}=i \omega \mu_{1 y} H_{y} \\
& E_{x}=\frac{i}{\omega \varepsilon_{1 x}} \frac{\partial H_{y}}{\partial z} \\
& E_{z}=-\frac{i}{\omega \varepsilon_{1 z}} \frac{\partial H_{y}}{\partial x}
\end{aligned}
$$

Where $\omega$ and $\beta$ denote the angular frequency and longitudinal propagation constant, respectively. In the cladding layer, three similar equations can be obtained by substituting corresponding cladding permittivity and permeability in Eq. (1a-1c). From above-mentioned equations, electromagnetic fields for even $T M$ modes in an anisotropic slab waveguide are written as:

$$
\begin{aligned}
& E_{z 1}=\frac{i k^{\prime}}{\omega \varepsilon_{1 z}} A \sin \left(k^{\prime} x\right) \text { - } \\
& H_{y 1}=A \cos \left(k^{\prime} x\right) \text { - }(x \leq d) \\
& E_{z 2}=\frac{x}{|x|} \frac{i \alpha}{\omega \varepsilon_{2}} A \cos \left(k^{\prime} d\right) e^{-\alpha(|x|-d)}-(2 \mathrm{a}) \quad(x \geq d) \\
& H_{y_{2}}=A \cos \left(k^{\prime} d\right) e^{-\alpha(|x|-d)}-(2 \mathrm{c}) \quad(x \geq d)
\end{aligned}
$$


Where subscripts 1and 2 stand for the core layer and cladding layer, respectively. A is the amplitude for magnetic fields in the core layer while $\alpha$ is the evanescent coefficient, which can be written as $\alpha=\sqrt{\beta^{2}-k_{0}{ }^{2} \varepsilon_{2} \mu_{2}}, k^{\prime}$ is the transverse wave number, which can be expressed as $k^{\prime}=\sqrt{\frac{\varepsilon_{1 z}}{\varepsilon_{1 y}}\left(k_{0}{ }^{2} \varepsilon_{1 y} \mu_{1 x}-\beta^{2}\right)}, k_{0}$ is the wave number in the vacuum. If $k^{\prime}$ is real for the oscillating modes while $k$ is imaginary for the surface modes. Besides, the time-and $z$ factor $e^{\mathrm{i}(\omega t-\beta z)}$ multiplying all the field components is omitted in all the equations.

Considering the continuous boundary conditions for the transverse electromagnetic fields, if $\varepsilon_{1 x}, \varepsilon_{1 y}$ and $\varepsilon_{1 z}$ are all negative, but $\mu_{1 x}$ is positive, after some algebraic manipulations, the dispersion equation for $T M$ surface modes is expressed as:

$$
k d-\tanh ^{-1}\left(\frac{\varepsilon_{1 z} \mid \alpha}{\varepsilon_{2} k}\right)=m \pi
$$

Where $k=\sqrt{\left|\frac{\varepsilon_{1 z}}{\varepsilon_{1 y}}\right|\left(k_{0}{ }^{2}\left|\varepsilon_{1 y}\right| \mu_{1 x}+\beta^{2}\right)}, m=0,1,2,----$. Besides, the Power flux transmitting along z-direction in the waveguide is calculated by the integral of Poynting vector. The total power flux $\left(P_{t o t}\right)$ is calculated as follows:

$$
P_{t o t}=\frac{\beta}{\omega} \int_{0}^{\infty} \frac{1}{\varepsilon_{i}}\left|H_{y i}\right|^{2} d x \quad(i=1,2)
$$

Substituting Eqs. (2a)——(2d) into Eq. (4), the power flux in the waveguide can be written as:

$$
P_{t o t}=\frac{\beta A^{2}}{2 \omega \varepsilon_{1 x} k}\left[k d+\sinh (k d) \cdot \cosh (k d)+\frac{\varepsilon_{1 x} k}{\varepsilon_{2} \alpha} \cosh ^{2}(k d)\right]
$$

\section{Reflection coefficient and transmission coefficient in a taper slab waveguide with metamaterials}

The schematic geometry of a taper slab waveguide with metamaterials is shown in Fig.2. The parameters are same as that in Fig.1 except the waveguide thickness. Similar to Ref. [12], we 
take a multi-step approximation method and obtain the reflection coefficient and transmission coefficient for $T M$ surface modes in a tapered slab waveguide with metamaterials:

$$
a_{r}=\frac{\beta_{1}-\beta_{2}}{\beta_{1}+\beta_{2}}
$$

$$
c_{t}=\frac{2 \beta_{1} \beta_{2}}{\omega P\left(\beta_{1}+\beta_{2}\right)} \int_{0}^{\infty} \frac{1}{\varepsilon_{i}} H_{y}^{(i)} H_{y}^{(t)} d x \quad(i=1,2)
$$

In this section, the subscript " 1 "expresses the quantities in the left hand side, while " 2 " is that in the right hand side. $H_{y}^{(i)}$ expresses input magnetic fields and $H_{y}^{(t)}$ means output magnetic fields in the waveguide. If the even $T M$ surface mode propagates in a taper slab waveguide, $H_{y}^{(t)}$ can be written as:

$$
H_{y}^{(t)}=H_{y 1}^{(t)}+\frac{1}{2}\left(\frac{\partial H_{y}^{(t)}}{\partial d_{2}}\right)_{d_{2}=d_{1}} \Delta d-
$$

Where $\Delta d=d_{2}-d_{1}$ is small. Instituting Eqs. (2a)--(2d) into Eq. (7), we can obtain:

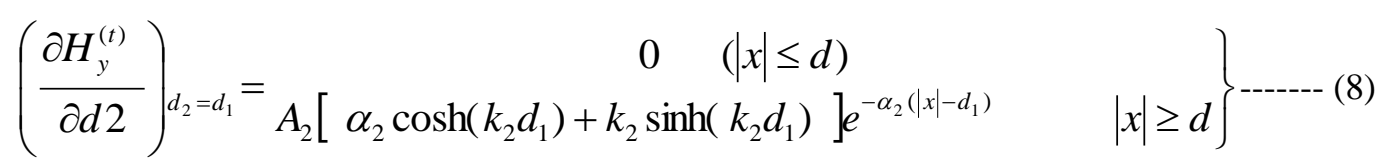

With the help of equations (7) and (8), considering the orthogonality of $T M$ modes, the transmission coefficient $c_{t}$ can be written as:

$c_{t}=\frac{\beta_{1} \beta_{2}}{\beta_{1}+\beta_{2}} \frac{A_{1} A_{2} \Delta d}{\omega \varepsilon_{2} P_{t o t}} \frac{\cosh \left(k_{1} d_{1}\right)}{\alpha_{1}+\alpha_{2}}\left[\alpha_{2} \cosh \left(k_{2} d_{1}\right)+k_{2} \sinh \left(k_{2} d_{1}\right)\right] \int_{0}^{L} e^{-i\left(\beta_{1}-\beta_{2}\right) z} d z$

Where $\mathrm{L}$ is the length of the taper waveguide and its waveguide wall is a line with slope $K$.

Therefore, Eq. (9) can be written as:

$c_{t}=\frac{2 \beta_{1} \beta_{2} K}{\beta_{1}+\beta_{2}} \frac{A_{1} A_{2}}{\omega \varepsilon_{2} P_{t o t}} \frac{\cosh \left(k_{1} d_{1}\right)}{\alpha_{1}+\alpha_{2}}\left[\alpha_{2} \cosh \left(k_{2} d_{1}\right)+k_{2} \sinh \left(k_{2} d_{1}\right)\right] \frac{\sin \left[\left(\beta_{1}-\beta_{2}\right) L / 2\right]}{\beta_{1}-\beta_{2}} e^{-i\left(\beta_{1}-\beta_{2}\right) L / 2}$

Substituting Eq. (5) into Eq. (10), the coefficient $c_{t}$ of the transmitted surface mode in a taper slab 
waveguide with anisotropic metamaterials is written as:

$$
c_{t}=\frac{2 \sqrt{\beta_{1} \beta_{2}}}{\beta_{1}+\beta_{2}} \frac{\varepsilon_{1 x}}{\varepsilon_{2}} \frac{\cosh \left(k_{1} d_{1}\right)}{\alpha_{1}+\alpha_{2}} \frac{K\left[\alpha_{2} \cosh \left(k_{2} d_{1}\right)+k_{2} \sinh \left(k_{2} d_{1}\right)\right]}{\sqrt{d_{1}+\frac{\sinh \left(k_{1} d_{1}\right) \cosh \left(k_{1} d_{1}\right)}{k_{1}}+\frac{\varepsilon_{1 x}}{\varepsilon_{2}} \frac{\cosh ^{2}\left(k_{1} d_{1}\right)}{\alpha_{1}}}} \frac{\sqrt{d_{2}+\frac{\sinh \left(k_{2} d_{2}\right) \cosh \left(k_{2} d_{2}\right)}{k_{2}}+\frac{\varepsilon_{1 x}}{\varepsilon_{2}} \frac{\cosh ^{2}\left(k_{2} d_{2}\right)}{\alpha_{2}}}}{\sqrt{\alpha_{2}}}
$$

$\frac{\sin \left[\left(\beta_{1}-\beta_{2}\right) L / 2\right]}{\beta_{1}-\beta_{2}} e^{-i\left(\beta_{1}-\beta_{2}\right) L / 2}$

From equations (6a) and (11), power loss of $T M$ surface modes in a taper slab waveguide with metamaterials will be calculated as:

$$
\frac{\Delta p}{p}=1-\left|a_{r}\right|^{2}-\left|c_{t}\right|^{2}
$$

\section{Numerical results}

We consider multi-layer structure metamaterials consisted of metal and dielectric. Assuming that metal it is silver and the dielectric is germanium. The optical properties of silver are described as Drude model, $\varepsilon_{m}=\varepsilon_{\infty}-\frac{\omega_{p}^{2}}{\omega^{2}-i \omega \gamma}$, with a background dielectric constant $\varepsilon_{\infty}=5$, plasmas frequency $\omega_{p}=1.38 \times 10^{16} \mathrm{rad} / \mathrm{s}$ and collision frequency $\gamma=5.07 \times 10^{13} \mathrm{rad} / \mathrm{s}[13]$. Besides, the permittivity of germanium $\varepsilon_{d}$ is 16. For each period of the multi-layer structure, the thickness of silver layer $l_{1} \mathrm{~nm}$ and the thickness of germanium layer $l_{2} \mathrm{~nm}$. Hence, the total thickness of the multi-layer structure for one period is $l_{1}+l_{2} n m$, and its filling ratio of metal is expressed by $f_{m}=l_{1} /\left(l_{1}+l_{2}\right)$. In this model, we suppose that the period of multi-layer $\left(l_{1}+l_{2}=10 \mathrm{~nm}\right)$ is much less than operation wavelength (i.e. frequency $500 \mathrm{THz}$, the wavelength $600 \mathrm{~nm}$ ), the multi-layer metamaterials can be treated as a homogeneous effective medium that obeys the Maxwell-Garnet theory [14].

$$
\varepsilon_{x}=\varepsilon_{y}=\varepsilon_{m} f_{m}+\left(1-f_{m}\right) \varepsilon_{d}
$$




$$
\varepsilon_{z}=\frac{\varepsilon_{m} \varepsilon_{d}}{\varepsilon_{d} f_{m}+\left(1-f_{m}\right) \varepsilon_{m}}
$$

However, the components of permeability are same, i.e. $\mu_{1 x}=\mu_{1 y}=\mu_{1 z}=1$. As frequency increases from $420 \mathrm{THz}$ to $520 \mathrm{THz}$, the real parts of permittivity along $\mathrm{x}, \mathrm{y}$ and $\mathrm{z}$ directions are all negative. Moreover, the imaginary parts are much smaller than the corresponding real parts. In this case, for $k^{\prime}=\sqrt{\frac{\varepsilon_{1 z}}{\varepsilon_{1 y}}\left(k_{0}{ }^{2} \varepsilon_{1 y} \mu_{1 x}-\beta^{2}\right)}$, the factor $\frac{\varepsilon_{1 z}}{\varepsilon_{1 y}}$ is positive, but the factor $k_{0}{ }^{2} \varepsilon_{1 y} \mu_{1 x}$ is negative and that result the factor $\left(k_{0}{ }^{2} \varepsilon_{1 y} \mu_{1 x}-\beta^{2}\right)$ can be written as $-\left(k_{0}{ }^{2}\left|\varepsilon_{1 y}\right| \mu_{1 x}+\beta^{2}\right)$. Therefore, we can deduce that $k^{\prime}$ is an imaginary number, and it can be written as $k^{\prime}=i k=i \sqrt{\frac{\mid \varepsilon_{1 z}}{\varepsilon_{1 y}} \mid\left(k_{0}{ }^{2}\left|\varepsilon_{1 y}\right| \mu_{1 x}+\beta^{2}\right)}$. Because the transverse wave number is an imaginary, surface modes propagate in this taper waveguide. Besides, we assume the cover and the substrate extend to infinity and that demands electromagnetic wave must decay as it propagates into them. (i.e. it demands $\beta>k_{0} \sqrt{\varepsilon_{2} \mu_{2}}$ )

\subsection{The dispersive properties of $T M$ surface modes in a taper slab waveguide with anisotropic metamaterial}

In order to investigate the influence of the anisotropic metamaterials for a taper slab waveguide, we assume that the permittivity and permeability are constants in the cover and substrate, i.e. $\varepsilon_{2}=2.25, \mu_{2}=1$. Besides, the frequency and metal filling ratio are fixed at $500 \mathrm{THz}$ and 0.8 , respectively. The propagation properties for $T M$ surface modes in a taper slab waveguide with metamaterials are discussed details in the following.

Through Eqs. (3), (13a) and (13b), the typical dispersive curves are plotted in Fig. 3. It is found that $T M$ surface modes have similar dispersive properties for different mode number. As mode number $m$ increases from 0 to 2 , the dispersive curves move to right and their cutoff 
waveguide thickness becomes larger. As waveguide thickness increases, effective refractive index has two different values which mean that the effective refractive index increases as waveguide thickness at lower branch of effective refractive index, but it decreases with waveguide thickness at higher branch of effective refractive index. This is because wave vector and energy flow have the same direction in common materials but opposite direction in the metamaterials. For the former, its energy flow is larger than that of the latter leading to a positive slope for its dispersive curve and it is called forward mode. However, as the former energy flow is smaller than the latter's, the dispersion curve slope is negative, and it is called backward mode. Usually, anti-direction energy flow values are different, which usually results in two modes propagating in the waveguide.

\subsection{Reflection coefficient of $T M_{0}$ surface modes in a taper slab waveguide with metamaterials.}

4.2.1 The dependence of reflection coefficient on metal filling ratio

Using equations (3), (6a) and (13), as frequency $\mathrm{f}=500 \mathrm{THz}$, the slope of the taper border $\mathrm{K}=0.002$, and $d_{1}=0.0153 \mu \mathrm{m}, d_{2}$ changed with $\mathrm{z}$, some reflection coefficient curves were plotted in Fig. 4 (the waveguide parameters are same as that in section 4.1). From these curves, it is found that:(1) As z increases, reflection coefficient has two different values. This is because two surface modes coexist in the waveguide. One is small and nearly positive zero, the other one increases monotonously as z. Firstly, it increases slowly with z, and then, it goes up quickly at larger $\mathrm{z}$ values. Especially, as waveguide length $\mathrm{L}=7.7 \mu \mathrm{m}$, its reflection coefficient is getting close to 0.98 . (2) As $f_{m}$ increases from 0.7 to 0.9 , the middle parts of curves move up and the reflection becomes stronger, but at the initial parts (i.e. zero) and ending parts (i.e. near 0.99 as 
$\mathrm{L}=7.7 \mu \mathrm{m}$ ) are kept at fixed values. This property for reflection coefficient near1.0 is similar to the results in Ref. [10] and it is believed that this could help us construct extra strong reflectors in the future.

\subsubsection{The dependence of reflection coefficient on frequency}

From Eqs. (3), (6a) and (13), as $f_{m}=0.8$, the typical reflection coefficient curves with different frequencies ( $\mathrm{f}=450,480$ and $510 \mathrm{THz}$ ) were plotted in Fig. 5 (the waveguide parameters were chosen as same as section 4.2.1). From these curves, it is found that: With the development of $\mathrm{z}$, reflection coefficient for each frequency has two different values. As frequency increases from $420 \mathrm{THz}$ to $510 \mathrm{GHz}$, for the taper waveguide with metamaterials, the reflection curves depending on the frequency behave similarly to the reflection curves depending on the metal filling ratios (see section 4.2.1, and Fig. 4)

\section{3 Transmission coefficient of $T M_{0}$ surface mode in a taper slab waveguide}

\section{with metamaterial}

4.3.1 The dependence of transmission coefficient on metal filling ratio

From Eqs. (3), (11) and (13), under different metal filling ratio $\left(f_{m}=0.7,0.8,0.9\right)$, when frequency $\mathrm{f}=500 \mathrm{THz}$ and, the typical curves of transmission coefficient depending on $\mathrm{z}$ were plotted in Fig. 6 (the waveguide parameters were chosen as same as section 4.1). From these curves, it is found that: (1) The transmission coefficient increases with $\mathrm{z}$ in a lower region. After that, it does not change obviously. (2) As $f_{m}$ varies from 0.7 to 0.9 , the curves move down and the transmission coefficient becomes smaller. Furthermore, the transmission coefficient is less than 0.1 as $f_{m}$ changes. It can be deduced that extra big energy loss may exist in the taper waveguide.

4.3.2 The dependence of transmission coefficient on frequency 
coefficient depending on $\mathrm{z}$ for different frequency were plotted in Fig. 7 (set the same waveguide parameters as in section 4.2.1). From these curves, it is found that: with the development of $z$, the transmission coefficient for different frequency increases monotonously in the lower region. And it only changes from 0 to 0.05 . It is shown that extra small transmission coefficient may exist and it causes extra big energy loss. The power loss will be discussed as follows in details.

\subsection{Power loss of $T M_{0}$ surface mode in a taper slab waveguide with metamaterial}

\subsubsection{Power loss dependence on metal filling ratio}

From Eqs. (3), (6a), (12) and (13), as frequency equals 500THz, the power loss curves for different $f_{m}$ were plotted in Fig. 8 (other waveguide parameters are chosen as same as that shown in section 4.1). From these curves, it is found that: (1) As $\mathrm{z}$ increases, power loss $\Delta p / p$ decreases. Firstly, it decreases slowly, then, it drops quickly with the increase of $\mathrm{z}$. As $L \leq 2 \mu m$, it is noted that $\Delta p / p \geq 0.98$. This is because that energy flow transmits back in metamaterials while it goes forward in common materials when wave vector moves ahead. Furthermore, for metamaterials, there should be stronger energy loss than that of common materials. It is believed that extra high power loss will benefit us to construct novel absorbers. (2) As $f_{m}$ increases from 0.7 to 0.9 , the curves move down. It means that power loss will be less. It seems difficult to understand. In fact, energy loss is caused by not only metal filling ratio, but also synthesized multi-layer structure.

\subsubsection{Power loss dependence on frequency}

Similar to section 4.2.2, as metal filling ratio $f_{m}=0.8$, when we choose the same waveguide parameters as section 4.2.1, the power loss curves under different frequency can be 
plotted as shown in Fig. 9. From these curves, it is found that: As frequency increases, the curves move down. It means that power loss will be less. However, as $L \leq 2 \mu m$, it is noted that $\Delta p / p \geq 0.99$ for different frequencies, the extra strong propagation loss has been verified in Ref. [15, 16] and it is also believed that the extra strong propagation loss could be used in designing novel absorbers in the future.

\section{Conclusions}

The TM surface mode and its propagation properties in a taper slab waveguide with anisotropic metamaterials in the core and common material in the claddings are investigated in details. From Maxwell's equations, we obtain some equations for the TM surface mode, such as: the dispersion, reflection coefficient, transmission coefficient and power loss equations. Subsequently, by numerical method, the characteristic curves of the dispersion depending on waveguide thickness for different mode number, and the curves of reflection coefficient, transmission coefficient and power loss depending on the $\mathrm{z}$ values for different metal filling ratios and frequencies are plotted. From these curves, it is found that:

(1) As mode number $m=0,1$ and 2, the effective refractive index has two different values which reveals a kind of double mode-degeneracy dispersion property;

(2) For different metal filling ratios $f_{m}(0.7,0.8$ and 0.9$)$, as frequency equals $500 \mathrm{THz}$, and waveguide length $\mathrm{L}=7.7 \mu \mathrm{m}$, the reflection coefficient is near 0.98 ; meanwhile, for different frequencies $(450 \mathrm{THz}, 480 \mathrm{THzand} 500 \mathrm{THz})$, as $f_{m}=0.8$ and waveguide length $\mathrm{L}=7.7 \mu m$, the reflection coefficient is near 0.99. Extra high reflection coefficient can be used to design extra strong reflectors.

(3) For different metal filling ratios $\left(f_{m}=0.7,0.8\right.$ and 0.9$)$, as $\mathrm{f}=500 \mathrm{THz}$, the transmission 
coefficient $\left|c_{t}\right|^{2} \leq 0.1$; meanwhile, for different frequencies (450THz, 480THzand 500THz), as $f_{m}=0.8,\left|c_{t}\right|^{2} \leq 0.05$. Extra small transmission coefficient may have extra big energy loss.

(4) For different metal filling ratios $\left(f_{m}=0.7,0.8\right.$ and 0.9$)$, as $\mathrm{f}=500 \mathrm{THz}$, the power loss $\Delta p / p \geq 0.98$; meanwhile, for different frequencies $(450 \mathrm{THz}, 480 \mathrm{THzand} 500 \mathrm{THz})$, as $f_{m}=0.8$ and $\Delta p / p \geq 0.99$. Extra high power loss means extra big energy loss in the waveguide. That will benefit us to design extra strong absorbers in the future.

\section{Acknowledgments:}

This work is supported by the National Natural Science Foundation of China (Grant No. 61275070); Zhejiang Provincial National Science Foundation China (Grant No.LY15A040003)

\section{References}

[1] V G Veselago. Electrodynamics of substances with simultaneously negative electrical and magnetic properties Sov Phyis USPEKHI 10 (1968) 509 - 517.

[2] D R Smith, W J Padilla, D C VIER, et al. Composite medium with simultaneously negative permeability and permittivity. Phys Rev Lett 84（2000） 4184-4187.

[3] I V Shadrivov, A A Sukhorukov, Y S Kivshar. Guided modes in negative-refractive-index waveguides Phys. Rev. E, 67 (2003) 057602.

[4] J L He, Y Jin, Z Hong, S L He. Slow light in a dielectric waveguide with negative-refractive-index photonic crystal cladding Opt. Exp. 16 (2008) 11077-11082

[5] Y He, J Zhang, C F Li. Guided modes in a symmetric five-layer left-handed waveguide J. Opt. Soc. Am. B 25 (2008) 2081-2091.

[6]S.M. Prokes, Orest J. Glembocki, J.E. Livenere et al. Hyperbolic and plasmonic properties of Silicon/Ag aligned nanowire arrays Opt. Exp. 21 (2013) 14962-14974 
[7] Christos Argyropoulos, Nasim Mohammadi Estakhri, Francesco Monticone, et al. Negative refraction, gain and nonlinear effects in hyperbolic metamaterials. Opt. Exp. 21 (2013) 15037-15047

[8] K. L.Tsakmakidis, A. D. Boardman, O.Hess, Trapped rainbow storage of light in metamaterials, Nature 450(2007) 397

[9] H.Hu, D. Ji and X. Zeng, et al. Rainbow Trapping in Hyperbolic Metamaterial Waveguide. Sci Rep. $3(2013) 1249$

[10] S. He, Y. He, and Y. Jin Revealing the truth about 'trapped rainbow' storage of light in metamaterials Sci. Rep. 2(2012) 583

[11]Andrei Andryieuski, V. SERGEI ZHUKOVSKY and V. ANDREI Lavrinenko1. Rough metal and dielectric layers make an even better hyperbolic metamaterial absorber Optics Express, 22(2014) $14975-14980$

[12] D. Marcuse Transmission Optics. Cheng Xi-wang transl. Beijing: Post \& Telecom Press, 1987.

[13]A. Sihvola Electromagnetic mixing formulas and applications. London: Institution of Electrical Engineers, 1999.

[14] P.B Johnson, R. W. Christy, Optical constants of the Nobel Metals Phys. Rev. B. 6(1972)43704379

[15] Guang-Yu Guo, Klimov Vasily, Shulin Sun, and Wei-Jin Zheng. Metamaterial slab-based super-absorbers and perfect nanodetectors for single dipole sources Opt. Exp. 21 (2013) 11338-11348

[16] Jing Zhou, F. Alexander Kaplan, et al. Experiment and Theory of the Broadband Absorption by a Tapered Hyperbolic Metamaterial Arrays . ACS Photonics, 1(2014) 618-624 


\section{FIGURE CAPTIONS:}

Fig. 1 Schematic geometry of a three-layer slab waveguide with metamaterials.

Fig. 2 Schematic geometry of a taper slab waveguide with metamaterials.

Fig. 3 Dispersion curves for $T M$ surface modes depend on mode number. The effective-refractive-index verses waveguide thickness as frequency is $500 \mathrm{THz}$. The core layer is anisotropic metamaterial as metal filling ratio is 0.8 . Both the cover and substrate are the same common material with dielectric permittivity $\left(\varepsilon_{1}=2.25\right)$ and magnetic permeability $\left(\mu_{1}=1.0\right)$

Fig. 4 The reflection coefficient of $T M_{0}$ mode verses electromagnetic wave travels along z-direction. It depends on metal filling ratios ( $f_{m}=0.7,0.8$ and 0.9 ), and the other corresponding parameters employed are the same as Fig. 3.

Fig. 5 The reflection coefficient of $T M_{0}$ mode verses electromagnetic wave travels along z-direction. It depends on frequency ( $f=450 \mathrm{THz}, 480 \mathrm{THz}$ and $500 \mathrm{THz})$, and the other corresponding parameters employed are the same as Fig. 3.

Fig. 6 The transmission coefficient of $T M_{0}$ mode verses electromagnetic wave travels along z-direction. It depends on metal filling ratios ( $f_{m}=0.7,0.8$ and 0.9 ), and the other corresponding parameters employed are the same as Fig. 3.

Fig. 7 The transmission coefficient of $T M_{0}$ mode verses electromagnetic wave travels along z-direction. It depends on frequency ( $f=450 \mathrm{THz}, 480 \mathrm{THz}$ and $500 \mathrm{THz}$ ), and the other corresponding parameters employed are the same as Fig. 3. 
Fig. 8 The power loss of $T M_{0}$ mode verses electromagnetic wave travels along z-direction. It depends on metal filling ratios $\left(f_{m}=0.7,0.8\right.$ and 0.9$)$, and the other corresponding parameters employed are the same as Fig. 3.

Fig. 9 The power loss of $T M_{0}$ mode verses electromagnetic wave travels along z-direction. They depend on frequency. The frequency is $450 \mathrm{THz}, 480 \mathrm{THz}$ and $500 \mathrm{THz}$, and the other corresponding parameters employed are the same as Fig. 3. 
1

2

3

4

5

6

7

9

10

11

12

13

14

15

16

17

18

19

20

21

22

23

24

25

26

27

28

29

30

31

32

33

34

35

36

37

38

39

40

41

42

43

44

45

46

47

48

49

50

51

52

53

54

55

56

57

58

59

60

61

62

63

64

65

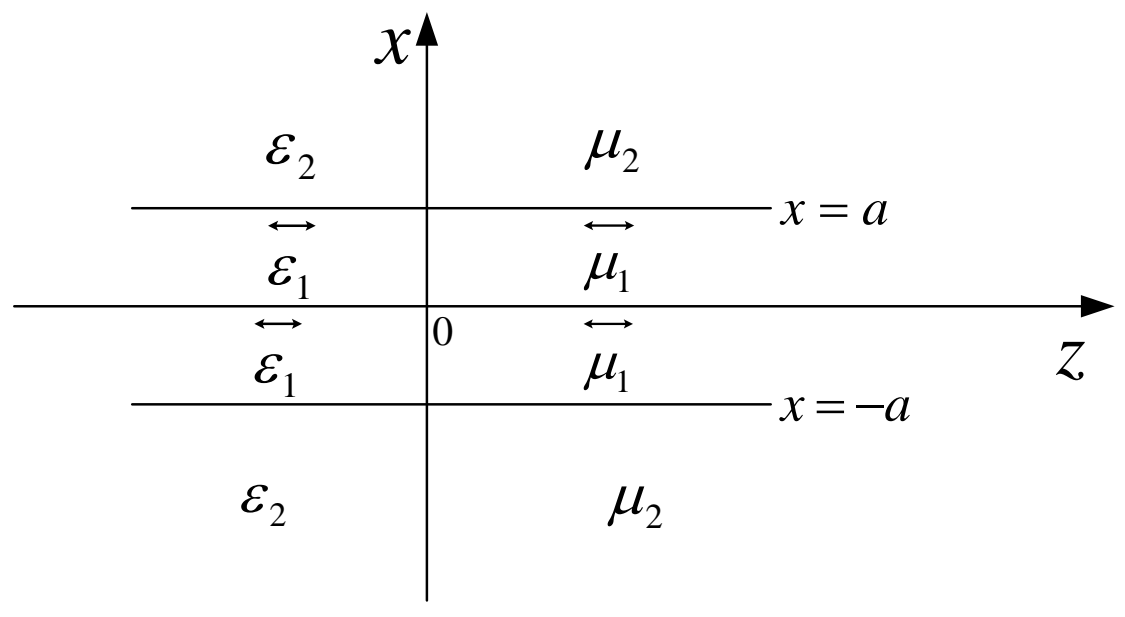

Fig. 1 
1

2

3

4

5

7

9

10

11

12

13

14

15

16

17

18

19

20

21

22

23

24

25

26

27

28

29

30

31

32

33

34

35

36

37

38

39

40

41

42

43

44

45

46

47

48

49

50

51

52

53

54

55

56

57

58

59

60

61

62

63

64

65

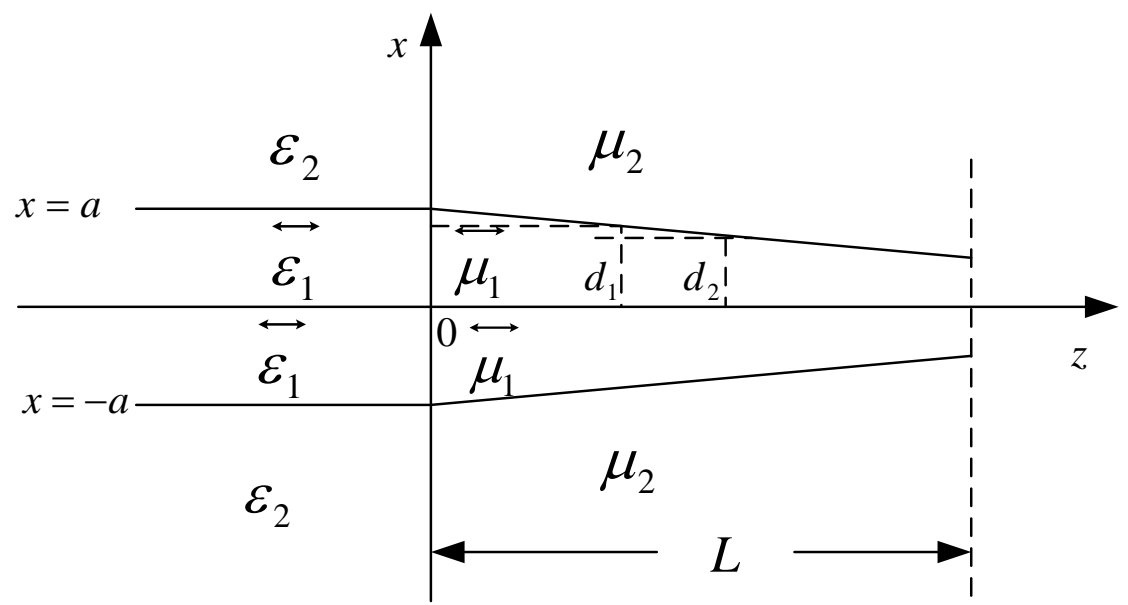

Fig. 2 


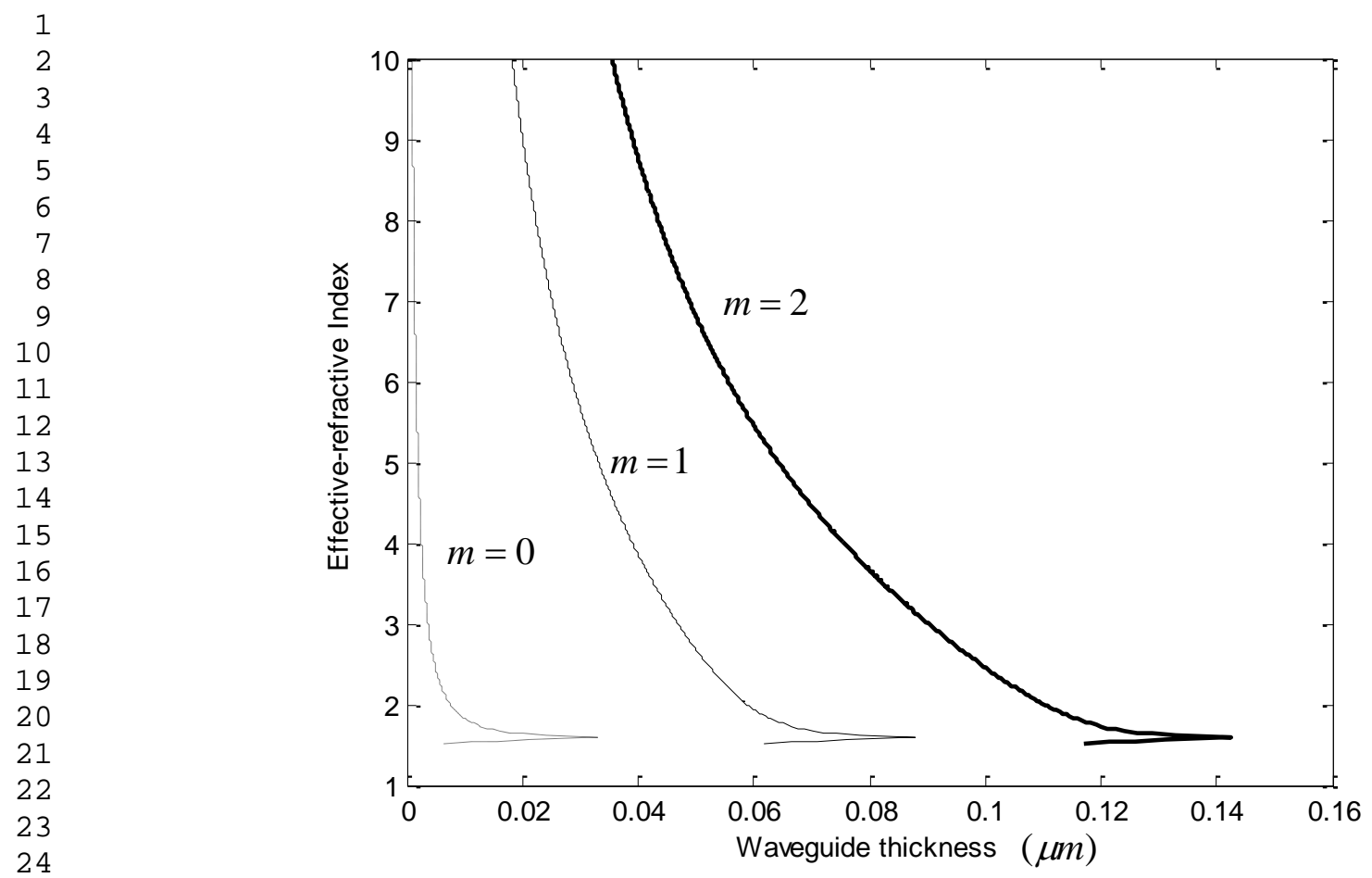

25

26

27

28

29

30

31

32

33

34

35

36

37

38

39

40

41

42

43

44

45

46

47

48

49

50

51

52

53

54

55

56

57

58

59

60

61

Fig. 3 


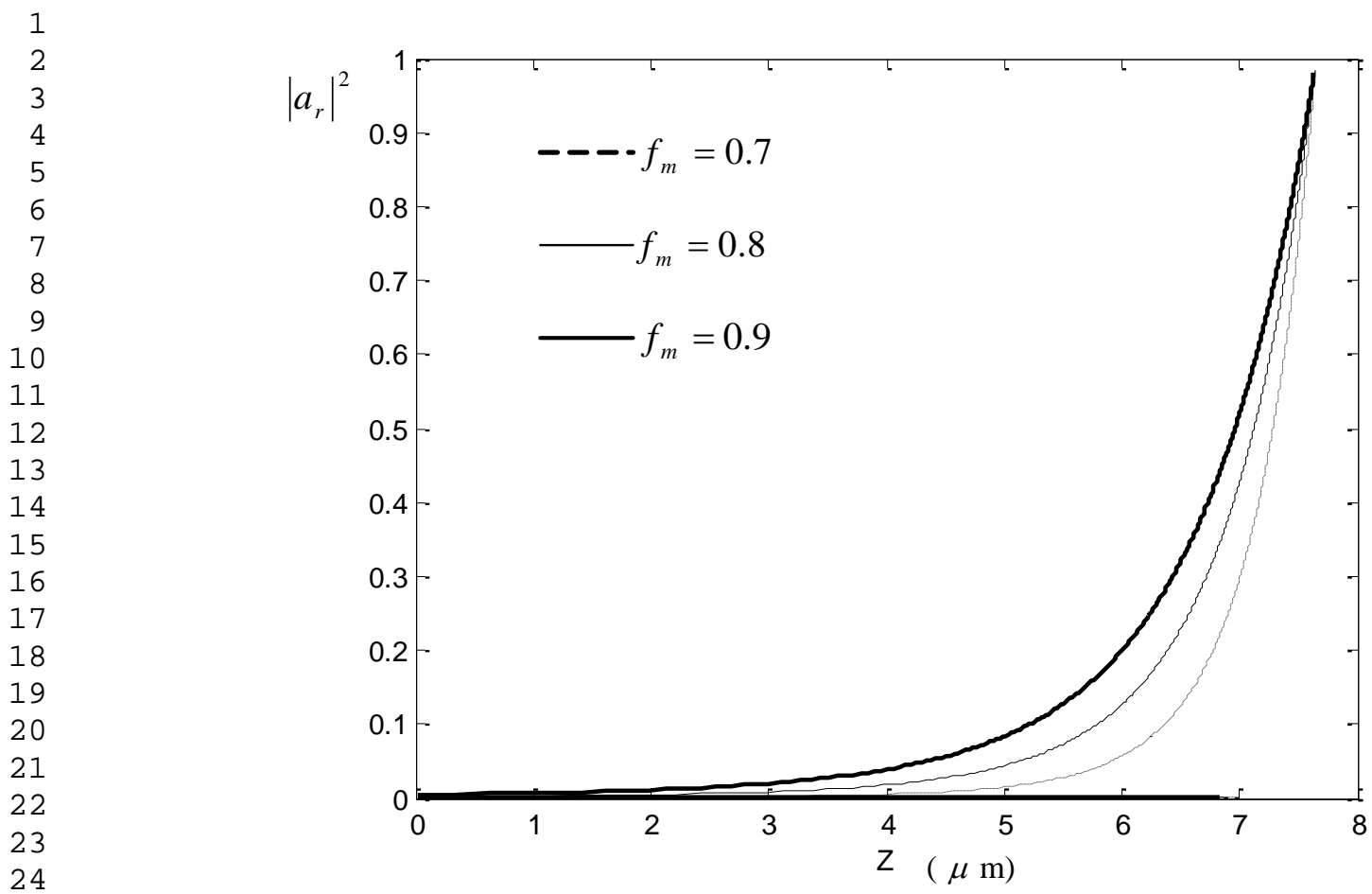

Fig. 4 
1

2

3

4

5

6

7

8

9
10

11

12

13

14

15

16

17

18

19

20

21

22

23

24

25

26

27

28

29

30

31

32

33

34

35

36

37

38

39

40

41

42

43

44

45

46

47

48

49

50

51

52

53

54

55

56

57

58

59

60

61

62

63

64

65

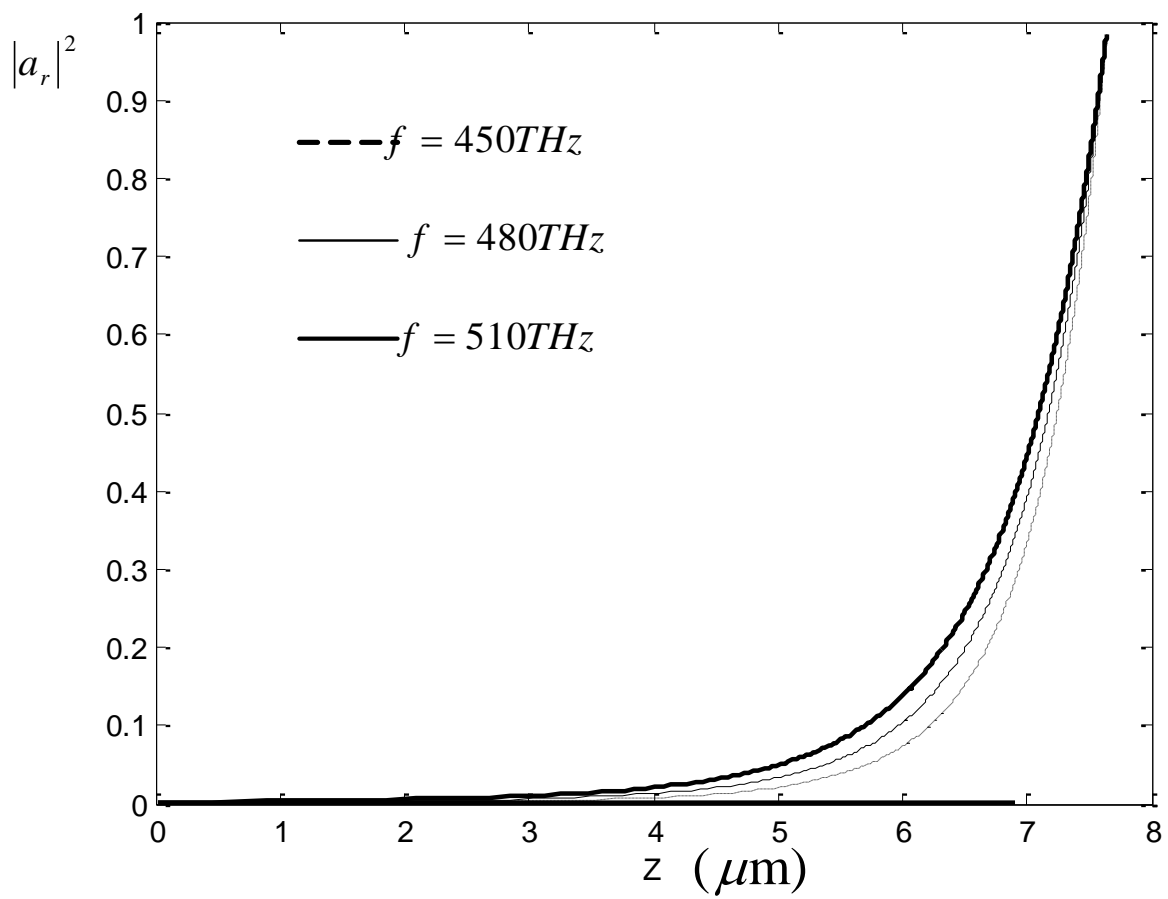

Fig. 5 
1

2

3

4

5

6

7

8

10

11

12

13

14

15

16

17

18

19

20

21

22

23

24

25

26

27

28

29

30

31

32

33

34

35

36

37

38

39

40

41

42

43

44

45

46

47

48

49

50

51

52

53

54

55

56

57

58

59

60

61

62

63

64

65

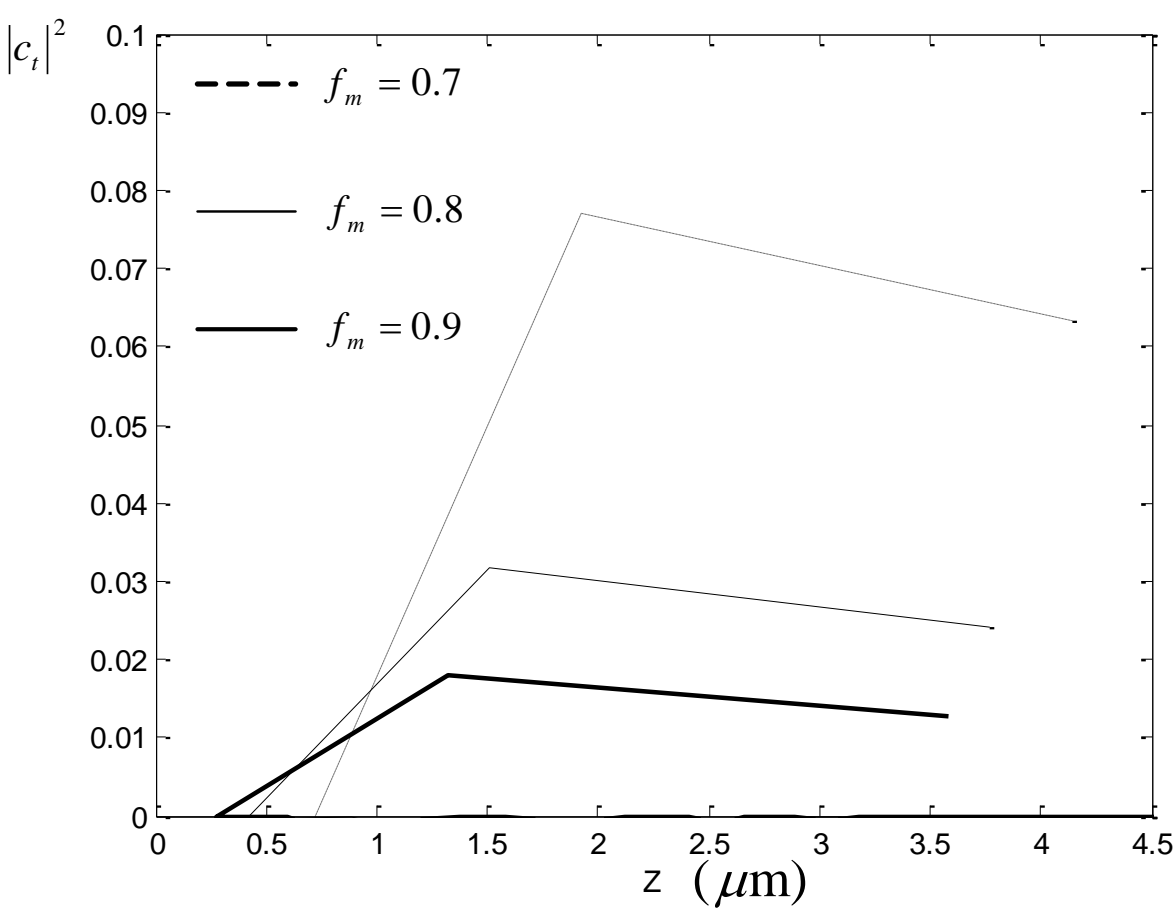

Fig. 6 
1

2

3

4

5

6

7

8

10

11

12

13

14

15

16

17

18

19

20

21

22

23

24

25

26

27

28

29

30

31

32

33

34

35

36

37

38

39

40

41

42

43

44

45

46

47

48

49

50

51

52

53

54

55

56

57

58

59

60

61

62

63

64

65

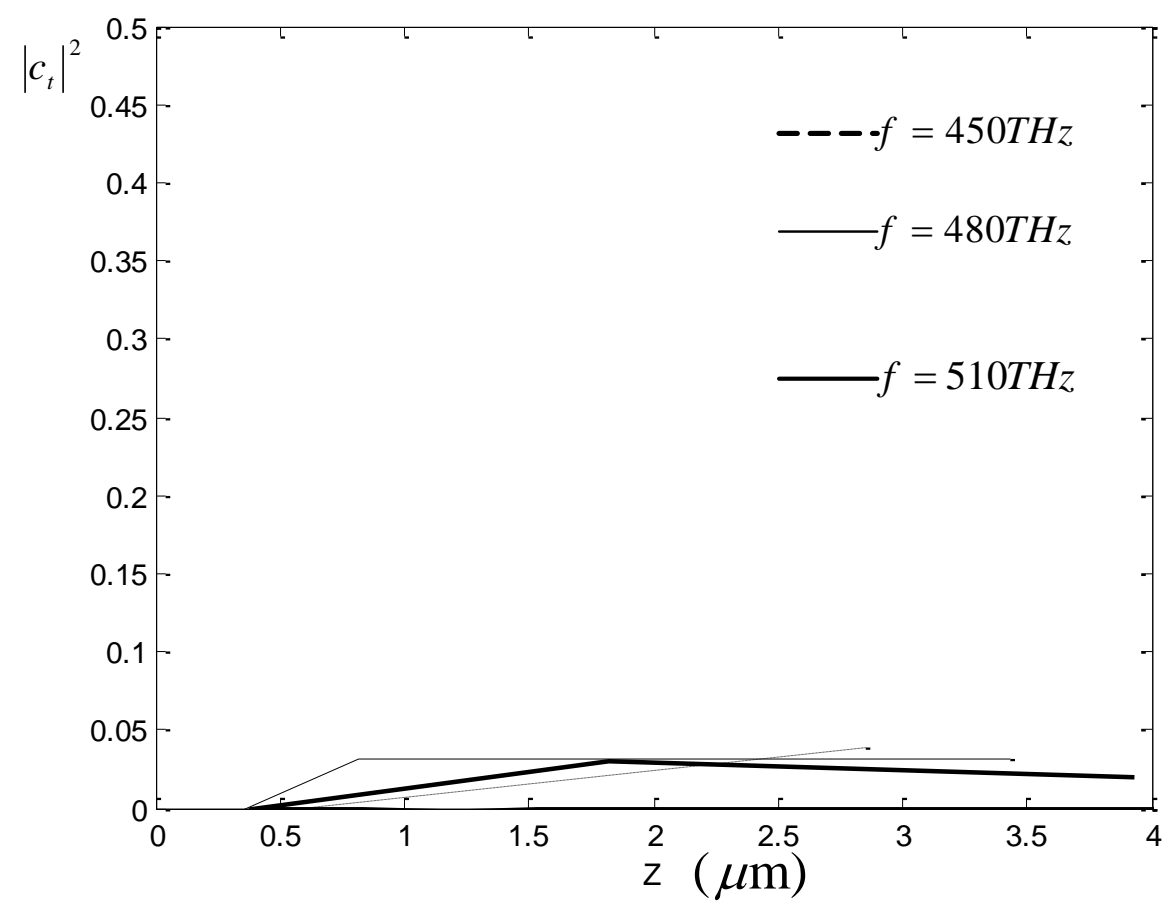

Fig. 7 


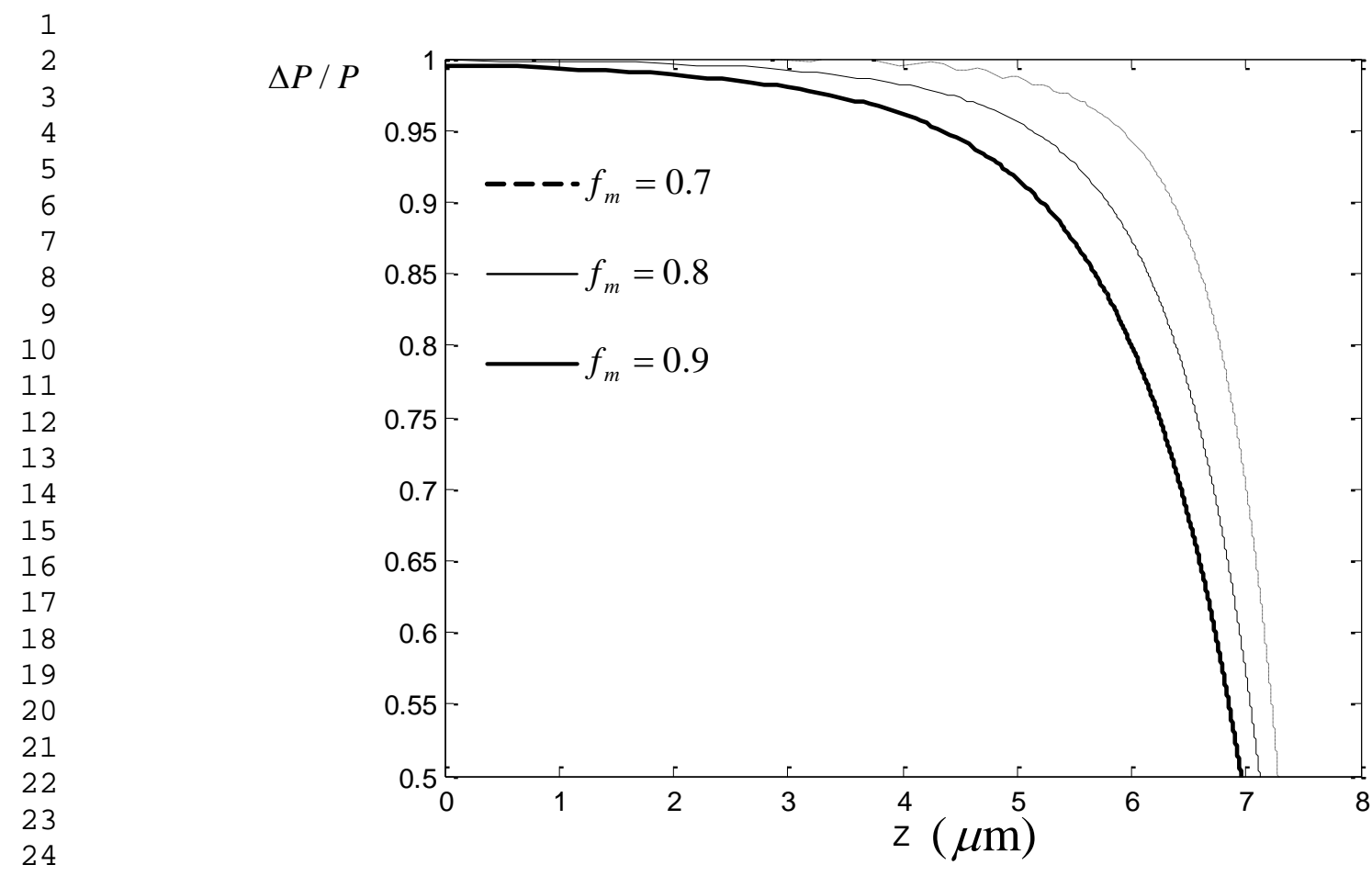

Fig. 8 


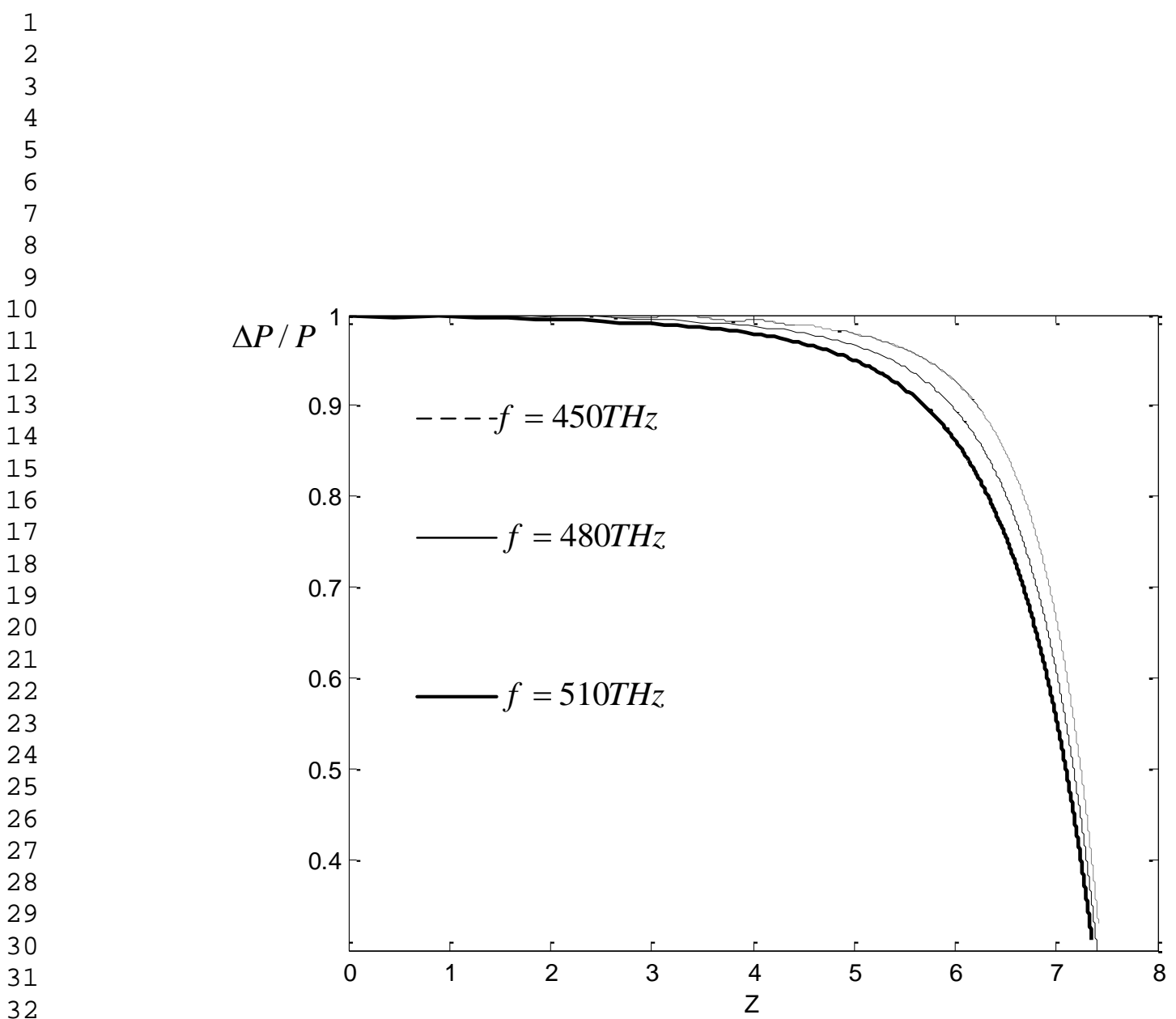

Fig. 9 